\title{
PROGRESOS de

\section{Efectos de un programa de ejercicio físico supervisado sobre la estructura cardiaca durante la gestación. Ensayo clínico aleatorizado}

\author{
María Perales $^{a, *}$, María Luaces $^{b}$, María Isabel Barriopedro $^{a}$, Rocío Montejo ${ }^{c}$ \\ y Rubén Barakat ${ }^{a}$
}

a Facultad de Ciencias de la Actividad Física y del Deporte (INEF), Universidad Politécnica de Madrid, Madrid, España

${ }^{\mathrm{b}}$ Servicio de Cardiología, Hospital Universitario de Fuenlabrada, Madrid, España

' Servicio de Ginecología y Obstetricia, Hospital Universitario de Fuenlabrada, Madrid, España

Recibido el 11 de julio de 2011; aceptado el 11 de enero de 2012

Accesible en línea el 22 de marzo de 2012

\section{PALABRAS CLAVE \\ Embarazo; \\ Ejercicio físico; \\ Sistema cardiovascular}

\section{KEYWORDS}

Pregnancy;

Physical exercise;

Cardiovascular system

\begin{abstract}
Resumen
Objetivo: Analizar la seguridad del ejercicio aeróbico para el sistema cardiovascular de las mujeres gestantes y valorar las posibles mejoras cardiovasculares producidas.

Sujetos y métodos: Se diseñó un ensayo clínico aleatorizado, no apareado, no enmascarado. El registro de datos se realizó en las semanas 20 y 34 de gestación. En la semana 20, se estudió a 45 gestantes del grupo de ejercicio (GE), de las cuales completaron el estudio 35. Del grupo de control (GC), en la semana 20 participaron 14, y completaron el estudio 9.

Resultados: En la semana 20 hubo mejoras en el índice de masa corporal (GE: 25,1 $\pm 4,3 / G C$ : $27,9 \pm 4,5)(p=0,04)$ y en la presión arterial sistólica (GE: 104,7 $\pm 10,9 / G C: 112,5 \pm 11,3)$ $(p=0,02)$. En el GC hubo presencia de hipertrofia concéntrica $(n=2,14,3 \%)$. En la semana 34, el GE presentó un mayor volumen sistólico (GE: 52,33 $\pm 10,42 / G C: 43,69 \pm 9,48)(p=0,03)$.

Conclusión: El ejercicio aeróbico durante el embarazo es seguro para el sistema cardiovascular, mejorando incluso algunos parámetros.

(c) 2011 SEGO. Publicado por Elsevier España, S.L. Todos los derechos reservados.
\end{abstract}

Effects of a supervised exercise program on cardiac structure during pregnancy. Randomized clinical trial Abstract
Objective: To analyze the safety of aerobic exercise for the cardiovascular system in pregnant
women, and to assess improvement in the cardiovascular system.
Subjects and methods: A randomized clinical trial was designed. Data collection was performed
at weeks 20 and 34 of gestation. At week 20,45 women were studied in the exercise (EG), of

\footnotetext{
* Autor para correspondencia.

Correo electrónico: maria.perales@upm.es (M. Perales).
} 
which 35 completed the study. There were 14 pregnant women in the control group (CG) in week 20, of which nine completed the study.

Results: At week 20 there were improvements in body mass index (EG: 25.1.土.4.3/CG: $27.9 \pm 4.5)(P=.04)$, and in systolic blood pressure (EG: 104.7 $\pm 10.9 / C G: 112.5 \pm 11.3$ ) $(P=.02)$. In the $C G$ there was concentric hypertrophy $(\mathrm{N}=2 ; 14.3 \%)$. At week 34 , the $\mathrm{EG}$ had a higher stroke volume (EG: $52.33 \pm 10.42 / \mathrm{GC}: 43.69 \pm 9.48)(P=.03)$.

Conclusion: Aerobic exercise during pregnancy is safe for the cardiovascular system and even improves some parameters.

(C) 2011 SEGO. Published by Elsevier España, S.L. All rights reserved.

\section{Introducción}

Un hecho fisiológico como es el proceso de embarazo y parto puede condicionar en gran medida la futura salud y calidad de vida de la mujer ${ }^{1}$; durante dicho proceso se producen diversas modificaciones que pueden agravarse con estilos de vida sedentarios. En este sentido, la remodelación del sistema cardiovascular, ocasionada en gran parte por los requerimientos fetoplacentarios ${ }^{2}$, requiere una especial atención.

En este sentido, son muchos los estudios que informan de posibles mejoras en la salud de estas gestantes a través de la práctica regular de ejercicio físico moderado durante el embarazo $^{3-10}$.

\section{Cambios cardiovasculares durante el embarazo}

A nivel cardiovascular se producen diversas modificaciones para garantizar la satisfacción de las nuevas demandas fetoplacentarias.

El corazón de la gestante, además de aumentar su tamaño, se desplaza hacia arriba, hacia la izquierda y gira sobre su eje longitudinal, como consecuencia de la progresiva elevación del diafragma por el incremento del tamaño del útero ${ }^{11,12}$.

El volumen sanguíneo se incrementa entre un 40-50\% respecto de su valor basal (aproximadamente $1.800 \mathrm{ml}$ ). Esto ocurre desde la semana 6-8 de gestación, alcanzando el valor máximo al final del segundo trimestre, donde se estabiliza ${ }^{10}$. La adaptación estructural del corazón necesaria para asumir este mayor volumen sanguíneo se traduce, en condiciones normales, en un incremento del diámetro telediastólico del ventrículo izquierdo (DTDVI) y mayor distensibilidad del ventrículo, con lo que se consigue el necesario aumento del gasto cardiaco sin elevar las presiones intracavitarias ni la presión sistémica ${ }^{2}$. Los diámetros diastólicos del ventrículo izquierdo aumentan hasta en un $6 \%$ y los del ventrículo derecho hasta en un $12 \%$. El grosor de la pared del ventrículo izquierdo también se incrementa con el embarazo.

El volumen sistólico aumenta progresivamente en el primer y el segundo trimestre. Yes hacia la mitad del embarazo cuando alcanza su valor máximo, siendo aproximadamente un $30 \%$ superior al de mujeres no gestantes. Al final del embarazo disminuye hasta recuperar los valores previos a la gestación ${ }^{13}$. No obstante, el volumen sistólico puede ser bastante inestable y está muy relacionado con las alteraciones del tamaño del corazón ${ }^{2}$.

La diferencia arterio-venosa de oxígeno se reduce considerablemente al inicio del embarazo ${ }^{14}$. Sin embargo, se mantiene un elevado aporte de oxígeno a los tejidos debido al aumento del gasto cardiaco, el volumen sistólico y la frecuencia cardiaca, siendo esta última fundamental al inicio del embarazo ${ }^{13}$.

La resistencia periférica en la circulación de la mujer embarazada se reduce significativamente debido a la circulación uteroplacentaria y a la modificación en la sensibilidad de las hormonas vasopresoras. Esta vasodilatación periférica mantiene la presión arterial en valores normales a pesar del aumento del volumen sanguíneo ${ }^{14}$, o incluso desciende en el primer trimestre, y alcanza su valor mínimo hacia la mitad del embarazo volviendo a recuperarse al final del mismo. Los cambios en la tensión sistólica son relativamente insignificantes ${ }^{15}$.

Seguramente uno de los aspectos más destacables de estas modificaciones es que solo se producen durante el periodo de embarazo $2,16,17$.

Por otra parte, con el ejercicio físico integrado en la vida cotidiana de gran parte de la población, encontramos que cada vez son más las gestantes interesadas en mantenerse físicamente activas durante el embarazo ${ }^{18,19}$, seguramente como un elemento que permita a la mujer embarazada mantener o aumentar su calidad de vida.

A pesar de que en los últimos años son muchos los estudios que se han ocupado de la relación entre el ejercicio físico y los resultados del embarazo $3,4,11,18$, permanecen ciertos interrogantes sobre la respuesta cardiovascular de la mujer gestante como respuesta a cargas moderadas y regulares de ejercicio físico.

El objetivo de este trabajo es conocer si la práctica de ejercicio regular, de carácter aeróbico moderado, es segura desde el punto de vista cardiovascular en las gestantes sanas y valorar en qué medida el ejercicio físico regular puede producir mejoras en la estructura cardiaca de estas mujeres.

\section{Sujetos y métodos}

\section{Diseño}

Se llevó a cabo un ensayo clínico, aleatorizado, no apareado y no enmascarado. El estudio fue aprobado por el Comité Ético de Investigación Clínica (CEIC) del Hospital de Fuenlabrada. Cada gestante firmó un consentimiento informado previo a su participación en el estudio.

\section{Muestra}

Inicialmente se contactó con 59 gestantes, todas pertenecientes a las consultas de obstetricia del Hospital Universitario de Fuenlabrada, de las cuales finalizaron el estudio 44. 


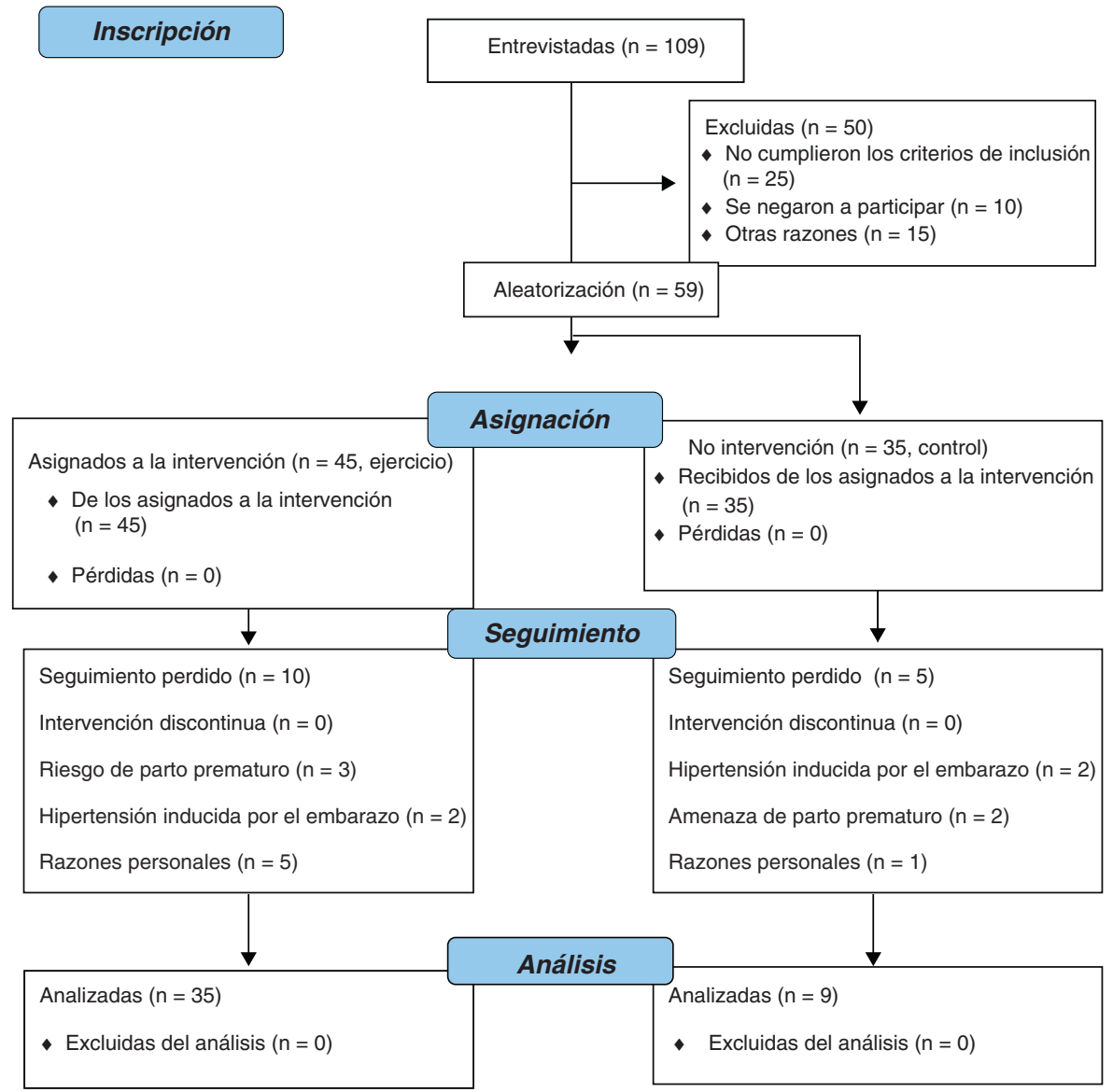

Figura 1 CONSORT 2010. Diagrama de flujo de las participantes en el estudio.

Inicialmente se contacto con 59 gestantes de las cuales finalizaron el estudio 44. Todas pertenecientes a las consultas de Obstetricia del Hospital Universitario de Fuenlabrada (fig. 1), siguiendo las líneas directrices del Consort Statement en su última actualización (www.consort-statement.org) que fue recientemente publicada ${ }^{20}$.

El registro de datos correspondientes a las variables de estudio se realizó en las semanas 20 y 34 de gestación. En la semana 20 se estudió a 45 gestantes pertenecientes al grupo de ejercicio (edad media $=31,3 \pm 3,3$ años) y 14 del grupo de control (edad media $=30,5 \pm 4,3$ años). En la semana 34 de gestación, continuaron el estudio 35 mujeres pertenecientes al grupo de ejercicio y 9 al grupo de control.

Todas las mujeres completaron una entrevista inicial en la que se obtuvieron datos de tipo personal y laboral (tabla 1).

\section{Criterios de inclusión}

El criterio de inclusión para formar parte de alguno de los grupos fue el de no presentar ningún tipo de contraindicación de carácter médico absoluto ${ }^{21}$, tales como: enfermedad de miocardio activa, insuficiencia cardiaca, enfermedad cardiaca reumática (clase II o superior), tromboflebitis, embolismo pulmonar reciente (últimos 5 años), enfermedad infecciosa aguda, incompetencia cervical, embarazo múltiple, hemorragia genital, rotura prematura de las membranas ovulares, crecimiento intrauterino retardado, macrosomía fetal, isoinmunización grave, enfermedad hipertensiva grave, ausencia de control prenatal, sospecha de sufrimiento fetal, riesgo de parto prematuro.

Tabla 1 Características maternas en ambos grupos de estudio

\begin{tabular}{lcll}
\hline Variables & $\begin{array}{l}\text { Grupo ejercicio } \\
\mathrm{n}=45\end{array}$ & $\begin{array}{l}\text { Grupo control } \\
\mathrm{n}=14\end{array}$ & $\mathrm{p}$ \\
\hline Edad (años) & $31,3 \pm 3,3$ & $30,5 \pm 4,3$ & 0,46 \\
$\begin{array}{l}\text { Paridad (n/\%) } \\
0\end{array}$ & $30 / 67$ & $9 / 69$ & \\
1 & $15 / 23$ & $5 / 31$ & 0,68 \\
Ocupación laboral (n/\%) & & \\
1 & $14 / 31$ & $6 / 42$ & 0,23 \\
2 & $13 / 29$ & $3 / 22$ & \\
3 & $18 / 40$ & $5 / 64$ & \\
Tabaquismo $(\mathrm{n} / \%)$ & & 0,41 \\
0 & $42 / 97$ & $13 / 93$ & \\
1 & $3 / 3$ & $1 / 7$ & \\
\hline
\end{tabular}

Paridad: 0 = ninguna gestación anterior. 1 = una o más gestaciones anteriores.

Ocupación laboral: $1=$ amas de casa. 2 = trabajo activo. 3 = trabajo sedentario.

Tabaquismo: 0 = no fumadora. 1 = fumadora. (Ambos durante el embarazo.) 


\section{Intervención. Programa de ejercicio físico}

El criterio básico seguido para la elaboración de las clases ha sido el de proporcionar a las gestantes un programa seguro y atractivo. Por ello, el diseño de este programa de ejercicio físico se apoyó en las líneas directrices del $\mathrm{ACOG}^{21}$, ya que se trata de unas líneas de actuación básicas y seguras mantenidas por casi la totalidad de los trabajos de investigación consultados ${ }^{11,14,21-25}$.

Todas las sesiones fueron desarrolladas en las instalaciones del Hospital Universitario de Fuenlabrada, en una sala ventilada, con luz natural, con música de apoyo, y con favorables condiciones ambientales (altitud alrededor de $600 \mathrm{~m}$, temperatura comprendida entre los 19 y los $21^{\circ} \mathrm{C}$ y una humedad relativa entre $50-60 \%$ ).

El desarrollo práctico de las sesiones contó con la guía y supervisión permanente de una licenciada en Ciencias de la actividad física y el deporte, y bajo la supervisión constante de un médico (obstetra) del Servicio de Ginecología y Obstetricia del Hospital, lo que ha resultado ser un factor de suma importancia.

\section{Características del programa}

El programa de ejercicio físico estuvo constituido por un total de 65 sesiones, con una frecuencia de 3 sesiones semanales, y una duración aproximada de 60 min cada una. La intensidad del trabajo se estableció a través de la fórmula de Karvonen ${ }^{26}$ entre un 55-60\% de la FCMx, y fue controlada por medio de pulsómetros Polar (FT60).

La incorporación al programa de ejercicio se realizó entre la semana 10-13 de gestación y la finalización del mismo fue hacia la semana 38-39 (salvo contraindicación de tipo obstétrico). Se les exigió un mínimo del $80 \%$ de adherencia al programa (52 sesiones).

\section{Estructura del programa}

Todas las sesiones se iniciaban con una entrada en calor de 7-8 min, compuesta por suaves desplazamientos y ejercicios de movilidad articular. A continuación, una parte central de 35-40 min, en la que se incluían tres tipos de trabajo:

1. El trabajo aeróbico, de mayor duración y caracterizado por su atractivo para estas mujeres.

2. El trabajo de fortalecimiento, con ejercicios de tonificación general de todo el cuerpo y otros dirigidos a aquellos grupos musculares más debilitados y necesitados en este proceso de embarazo.

3. Fortalecimiento de la estructura del suelo pélvico, siguiendo la propuesta de $\mathrm{Kegel}^{21}$.

Por último, una vuelta a la calma de 7-8 min, en la que se producía una bajada gradual de la intensidad de trabajo con ejercicios de flexibilidad y relajación.

\section{Variables del estudio y fuentes de recogida}

El estudio se realizó empleando aparatos General Electric (Fairfield, CT, EE. UU.): electrocardiógrafo, ecocardiógrafo Vivid-i y estación de trabajo Echopack 8. A todas las gestantes se les realizó un ecocardiograma transtorácico empleando un equipo comercial equipado con una sonda ultrasonográfica de 2,5 MHz (Vivid-i, GE Healthcare, Waukessha, WI, EE. UU.).

- Análisis estructural: mediciones convencionales de espesores parietales según las recomendaciones de la American Society of Echocardiography.

- Determinación del patrón geométrico ventricular izquierdo:

Normal: IMVI normal, GPR normal.

Remodelado concéntrico: IMVI normal, GPR aumentado. Hipertrofia concéntrica: IMVI aumentado, GPR aumentado.

Hipertrofia excéntrica: IMVI aumentado, GPR normal.

Además, se midieron el peso y la talla de cada una de las gestantes, tanto en la semana 20 como en la 34, para calcular el índice de masa corporal (IMC).

\section{Análisis estadístico}

El programa utilizado para el análisis de datos fue el Statistical Package for the Social Science (SPSS) versión 15.0.

Para el análisis de variables continuas (IMC, presión arterial y otros parámetros referentes a la distensibilidad del corazón), se realizó una comparación de medias con la técnica de la $\mathrm{t}$ de Student para muestras independientes. El tamaño del efecto fue calculado mediante la d de Cohen. En los casos en los que no se hallaron diferencias significativas, se calculó la potencia del contraste.

Para analizar la variable categórica de adaptación estructural del corazón, se utilizó la prueba de la chi al cuadrado de Pearson a partir del método Monte Carlo de estimación. Para las comparaciones a posteriori, se calcularon los residuos tipificados corregidos.

\section{Resultados}

Como se puede observar en la tabla 1, ambos grupos presentan similares características maternas en lo relativo a edad, paridad, ocupación laboral y tabaquismo.

\section{Semana 20 de gestación}

Las gestantes que realizaron ejercicio físico presentaron niveles menores de IMC en la mitad del embarazo (tabla 2) que las gestantes del grupo de control $(p=0,04)$, siendo el tamaño del efecto moderado ${ }^{27}$.

Asimismo, los valores de presión arterial sistólica (PAS) en la mitad del embarazo (tabla 2) fueron inferiores en las gestantes del grupo de ejercicio $\left(t_{58}=2,28 ; p=0,02\right)$. El tamaño del efecto fue moderado-alto $(d=0,72)$.

Atendiendo a la adaptación estructural del corazón (tabla 3), los resultados muestran que el patrón geométrico (relación espesor parietal/masa del ventrículo izquierdo) estuvo conservado con mayor frecuencia en las mujeres del GE $(N=38 ; 84,4 \%$, ) que en las del GC $(N=11 ; 78 \%)$. Sin embargo, solo se encontró una diferencia significativa en la hipertrofia concéntrica, ya que estuvo presente en 2 gestantes $(14,3 \%)$ del GC, siendo nula la presencia de este cuadro entre las gestantes del $\mathrm{GE}(\mathrm{N}=0)$. 
Tabla 2 Parámetros cardiovasculares e IMC. Semana 20 de gestación

\begin{tabular}{lcccc}
\hline Semana 20 & $\mu$ Grupo ejercicio $(\sigma)$ & $\mu$ Grupo control $(\sigma)$ & $p$ & d de Cohen \\
& $N=45$ & $N=14$ & & Potencia \\
\hline IMC (kg/m2) & $25,1 \pm 4,3$ & $27,9 \pm 4,5$ & $0,04^{\mathrm{a}}$ & $-0,66$ \\
PAS (mmHg) & $104,7 \pm 10,9$ & $112,5 \pm 11,3$ & $0,02^{\mathrm{a}}$ & $-0,72$ \\
PAD (mmHg) & $61,9 \pm 7,5$ & $66,2 \pm 8,6$ & 0,07 & 0,43 \\
VS (ml/lat) & $52,01 \pm 11,67$ & $50,03 \pm 10,75$ & 0,57 & 0,085 \\
DTDVI (cm) & $4,5 \pm 0,4$ & $4,5 \pm 0,3$ & 0,81 & 0,056 \\
DTSVI (cm) & $2,7 \pm 0,4$ & $2,8 \pm 0,3$ & 0,58 & 0,085 \\
SIV (cm) & $0,8 \pm 0,1$ & $0,7 \pm 0,1$ & 0,37 & 0,14 \\
PP (cm) & $0,7 \pm 0,1$ & $0,8 \pm 0,1$ & 0,17 & 0,27 \\
\hline
\end{tabular}

DTDVI: diámetro telediastólico del ventrículo izquierdo; DTSVI: diámetro telesistólico del ventrículo izquierdo; IMC: índice de masa corporal; PAD: presión arterial diastólica; PAS: presión arterial sistólica; PP: pared posterior; SIV: septo interventricular; PP: pared posterior. Datos expresados como valor \pm desviación estándar.

a Significativo al nivel 0,05 .

Tabla 3 Adaptación estructural del corazón en ambos grupos de estudio. Semana 20 de gestación

\begin{tabular}{llcl}
\hline Semana 20 & Grupo ejercicio $n(\%)$ & Grupo control $\mathrm{n}(\%)$ & $\mathrm{p}$ \\
\hline Patrón normal & $38(84,4)$ & $11(78,6)$ & 0,047 \\
Remodelado concéntrico & $7(15,6)$ & $1(7,1)$ \\
Hipertrofia concéntrica & 0 & $2(14,3)$ \\
Hipertrofia excéntrica & 0 & 0 & \\
\hline
\end{tabular}

\section{Semana 34 de gestación}

Como se puede observar en la tabla 4, los valores del DTDVI obtenidos en el GE son menores con respeto a los del GC $\left(\mathrm{t}_{43}=\right.$ $2,47 ; p=0,01)$. El tamaño del efecto fue grande $(d=-1,07)$.

El ejercicio físico tuvo un efecto positivo en el volumen sistólico (tabla 4), ya que en las mujeres del GE fue significativamente superior $\left(t_{37}=2,22 ; p=0,03\right)$. El tamaño del efecto fue grande $(d=0,86)$.

En la semana 34 de gestación, no se encontraron diferencias significativas en relación con la adaptación estructural del corazón (tabla 5).

\section{Discusión}

Este estudio ha tenido como objeto conocer en qué medida un programa de ejercicio físico puede coexistir con la realidad del proceso de embarazo e incluso mejorar ciertos parámetros referentes a la estructura cardiaca.

El volumen sistólico suele incrementarse durante el embarazo $^{2,13}$; este incremento suele ser mayor, tanto en reposo como durante el ejercicio, si la gestante es físicamente activa $^{28-30}$

El diámetro del ventrículo izquierdo, al igual que el entrenamiento aeróbico, produce incrementos en la población en general ${ }^{31}$; también se incrementa en las mujeres gestantes físicamente activas en comparación con las sedentarias, tanto en reposo como durante el ejercicio ${ }^{32-37}$. El hecho de que tanto el volumen sistólico como la contractilidad ventricular puedan incrementarse durante el ejercicio indica que el ventrículo es capaz de responder adecuadamente al estrés hemodinámico ${ }^{37}$.

Nuestros resultados en relación con el volumen sistólico coinciden con ciertos autores ${ }^{13,28-30}$, ya que en las gestantes del GE es significativamente mayor que en las gestantes del GC. Además, encontramos diferencias significativas en el

Tabla 4 Parámetros cardiovasculares e IMC. Semana 34 de gestación

\begin{tabular}{|c|c|c|c|c|c|}
\hline Semana 34 & $\mu$ grupo ejercicio $(\sigma) \mathrm{N}=35$ & $\mu$ Grupo control $(\sigma) N=9$ & $\mathrm{p}$ & $\mathrm{d}$ de Cohen & Potencia \\
\hline IMC $\left(\mathrm{kg} / \mathrm{m}^{2}\right)$ & $28,2 \pm 5$ & $30,2 \pm 4,8$ & 0,27 & & 0,18 \\
\hline PAS (mmHg) & $105,3 \pm 19,6$ & $108,3 \pm 9,6$ & 0,66 & & 0,072 \\
\hline PAD (mmHg) & $62,9 \pm 5,8$ & $65 \pm 8,6$ & 0,27 & & 0,19 \\
\hline VS (ml/lat) & $52,33 \pm 10,42$ & $43,69 \pm 9,48$ & $0,032^{a}$ & 0,86 & \\
\hline DTDVI $(\mathrm{cm})$ & $4,6 \pm 0,4$ & $5 \pm 0,3$ & $0,01^{\mathrm{a}}$ & $-1,07$ & \\
\hline DTSVI (cm) & $2,8 \pm 0,3$ & $3 \pm 0,3$ & 0,24 & & 0,21 \\
\hline $\operatorname{SIV}(\mathrm{cm})$ & $0,9 \pm 0,1$ & $0,8 \pm 0,1$ & 0,26 & & 0,20 \\
\hline $\mathrm{PP}(\mathrm{cm})$ & $0,8 \pm 0,1$ & $0,8 \pm 0,1$ & 0,96 & & 0,05 \\
\hline
\end{tabular}

Los datos se presentan como media \pm desviación típica.

a Significativo al nivel 0,05 . 
Tabla 5 Adaptación estructural del corazón en ambos grupos de estudio. Semana 34 de gestación

\begin{tabular}{lcc}
\hline Semana 34 & Grupo ejercicio $\mathrm{n} \mathrm{( \% )}$ & Grupo control N (\%) \\
\hline Patrón normal & $24(69,7)$ & $6(66,7)$ \\
Remodelado concéntrico & $9(24,2)$ & $1(11,1)$ \\
Hipertrofia concéntrica & $2(6,1)$ & $2(22,2)$ \\
Hipertrofia excéntrica & 0 & 0 \\
\hline
\end{tabular}

DTDVI siendo mayor en el GC que en el GE. Esto se debe a que el GE ha desarrollado un mayor espesor parietal (pared posterior y septo interventricular), lo que probablemente refleja un incremento en la función sistólica ya que hemos encontrado un aumento en el volumen sistólico en las mujeres que realizan ejercicio en la semana 34 de gestación.

La relevancia de las adaptaciones geométricas del ventrículo izquierdo está en relación con el reparto del estrés parietal. En poblaciones hipertensas o de edad avanzada, el aumento de espesores junto con diámetros cavitarios pequeños se asocia a mala elasticidad cardiaca (por tanto, son peores los patrones de remodelado concéntrico e hipertrofia excéntrica). Sin embargo, en nuestro grupo de estudio hemos encontrado que la función sistólica no solo se conserva sino que mejora, lo que refleja la adaptabilidad y preservación de propiedades elásticas de la fibra miocárdica en estas mujeres.

Con relación al comportamiento de la presión arterial, es importante aclarar que el embarazo es una condición fisiológica saludable, pero en determinadas situaciones puede existir un cierto riesgo de hipertensión para la mujer gestante ${ }^{38}$. Esta lleva consigo otra serie de complicaciones, como puede ser la preeclampsia, muy peligrosa ya que es la responsable del $22 \%$ de las muertes maternas ${ }^{39}$ y del $18 \%$ de las de los partos prematuros ${ }^{40-43}$; además, incrementa el riesgo de futuras enfermedades cardiovasculares ${ }^{44,45}$

El riesgo de desarrollar estas patologías aumenta si la mujer gestante presenta excesiva ganancia de peso, sobrepeso u obesidad $^{46-48}$, demostrando la estrecha relación entre factores metabólicos y cardiovasculares durante el embarazo.

Diversos estudios afirman que la actividad física puede disminuir potencialmente el riesgo a desarrollar hipertensión e incluso disminuir los valores de presión arterial en aquellas gestantes que realizan ejercicio físico regular ${ }^{49-51}$.

Nuestros resultados están en consonancia con ellos, ya que tanto el índice de masa corporal como la PAS han sido significativamente inferiores en el GE que en el GC en la semana 20 de gestación.

Respecto de la adaptación estructural del corazón con el ejercicio, los resultados de nuestro estudio afirman que tan solo hay diferencias significativas en la semana 20 de gestación, en la que hay más mujeres del GC que presentan una hipertrofia concéntrica. Conservándose, por tanto, un patrón normal en un mayor número de gestantes del grupo de ejercicio. Sin embargo, se puede observar como en la semana 34 hay un mayor porcentaje de mujeres del GE que presentan otro patrón geométrico diferente del normal, mientras que en el GC estos porcentajes permanecen más estables. Este cambio en la distribución de patrones geométricos es el resultado de la adaptación cardiaca al mayor volumen sistólico que manejan las mujeres del grupo de ejercicio.

Todos nuestros resultados cardiovasculares se encuentran dentro de los valores considerados normales para gestantes sanas $^{52}$.
Muchas de las variables obtenidas en el estudio, tales como pared posterior, septo interventricular, fracción de eyección del ventrículo izquierdo, son difíciles de discutir ya que existen pocos estudios que atiendan a dichos parámetros en gestantes que realizan ejercicio físico regular durante el embarazo.

\section{Limitaciones del estudio}

El pequeño tamaño de la muestra ha sido nuestro principal limitante para obtener diferencias estadísticamente significativas entre ambos grupos. Esto se ve reflejado en las bajas potencias de contraste obtenidas en el análisis estadístico de todas las variables estudiadas.

\section{Conclusión}

La participación en un programa de ejercicio aeróbico durante el embarazo es seguro desde el punto de vista cardiovascular, mejorando incluso algunos de los parámetros referentes a la estructura cardiaca.

\section{Financiación}

María Perales Santaella es beneficiaria de una beca de investigación homologada para la realización del Doctorado financiada por la UPM.

\section{Conflicto de intereses}

Los autores declaran no tener ningún conflicto de intereses.

\section{Agradecimientos}

Los autores de este trabajo quieren expresar su más sincero agradecimiento a los Servicios de Ginecología y Obstetricia, y Cardiología del Hospital Universitario de Fuenlabrada.

\section{Bibliografía}

1. Gouveia R, Martins S, Sandes A, Nascimento C, Figueira J, Valente $S$, et al. Pregnancy and physical exercise: myths, evidence and recommendations. Acta Med Port. 2007;20:209-14.

2. Artal R, Wiswell R, Drinkwater B. Exercise in pregnancy. 2nd ed. Baltimore: Williams and Wilkins; 1991.

3. Barakat R, Ruiz J, Stirling J, Zakynthinaki M, Lucia A. Type of delivery is not affected by light resistance and toning exercise training during pregnancy: a randomized controlled trial. Am J Obstet Gynecol. 2009;201:590. e1-6. 
4. Barakat R, Stirling J, Lucia A. Does exercise training during pregnancy affect gestational age? A randomised, controlled trial. Brit J Sport Med. 2008;42:674-8.

5. CavalcanteS, Cecatti J, Pereira R, Baciuk E, Bernardo A, Silveira C. Water aerobics II: maternal body composition and perinatal outcomes after a program for low risk pregnant women. Reprod Health. 2009;6:1.

6. Gavard JA, Artal R. Effect of exercise on pregnancy outcome [review]. Clin Obstet Gynecol. 2008;51:467-80.

7. Kinnunen T, Pasanen M, Aittasalo M, Fogelholm M, Hilakivi-Clarke L, Weiderpass $E$, et al. Preventing excessive weight gain during pregnancy - a controlled trial in primary health care. Eur J Clin Nutr. 2007;61:884-91.

8. Lombard C, Deeks A, Ball K, Jolley D, Teede H. Weight, physical activity and dietary behavior change in young mothers: short term results of the HeLP-her cluster randomized controlled trial. Nutr J. 2009;1:8-17.

9. Mottola M. Exercise prescription for overweight and obese women: pregnancy and postpartum. Obstet Gynecol Clin North Am. 2009;36:301-16.

10. Mottola MF, Giroux I, Gratton R, Hammond JA, Hanley A, Harris S, et al. Nutrition and exercise prevent excess weight gain in overweight pregnant women. Med Sci Sports Exerc. 2010;42:265-72.

11. Barakat R. El ejercicio físico durante el embarazo. Madrid: Pearson Alhambra; 2006.

12. Kisner C, Colby LA. Ejercicio terapéutico: fundamentos y técnicas. Barcelona: Editorial Paidotribo; 2005.

13. Walters WAW, MacGregor WG, Hills M. Cardiac output at rest during pregnancy and the puerperium. Clin Sci. 1966;3:0-1.

14. Olson D, Sikka RS, Hayman J, Novak M, Stavig C. Exercise in pregnancy. Curr Sports Med Rep. 2009;8:147-53.

15. MacGillivray I, Rose GA, Rowe B. Blood pressure survey in pregnany. Clin Sci. 1969;37-395.

16. Hytten F, Chamberlerlein G. Clinical Psysiology in Obstetrics. Oxford: Blackwell Scientific Publications; 1980.

17. Mottola $M$, Campbell $M$. Activity patterns during pregnancy. Can J Appl Physiol. 2003;28:642-53.

18. Barakat R, Lucia A, Ruiz J. Resistance exercise training during pregnancy and newborn's birth size: a randomised controlled trial. Int J Obes (Lond). 2009;33:1048-57.

19. Barakat R, Ruiz J, Lucia A. Exercise during pregnancy and risk of maternal anaemia: a randomised, controlled trial. $\mathrm{Br} \mathrm{J}$ Sports Med. 2009;43:954-6.

20. Schulz KF, Altman DG, Moher D, for the CONSORT Group. Consort 2010 Statemente:updated guidelines for reporting parallel group randomised trials. BMC Medicine. 2010;8:18.

21. ACOG. American College of Obstetricians and Gynecologists. Exercise during pregnancy and the postpartum period. Committe Opinion N. ${ }^{\circ}$ 267. Washington, DC. Obstet Gynecol. 2002;99: 171-3.

22. Mata F, Chulvi I, Roig J, Heredia J, Isidro F, Benítez J, et al. Prescripción del ejercicio físico durante el embarazo. Rev Andal Med Deporte. 2010;3:68-79.

23. Artal R. Ejercicio y embarazo. Barcelona: Ediciones Médici; 1995

24. Cowlin F. Women's fitness program development. Champaing: Human Kinetics; 2002.

25. Katja M, Kelly R, Fang W, Amy H, Aimee M. Physical activity patterns during pregnancy. Med Sci Sports Exerc. 2008;40: 1901-8.

26. Colbert S. Diabetes y ejercicio. Madrid: Ed. Tutor; 2003.

27. Cohen J. Quantitative methods in psychology. A power primer. Psychol Bull. 1992;112:155-9.

28. Morton MJ, Paul NS, Compos GR, Hartz MV, Metcalfe J. Exercise dynamics in late gestation: effects of physical training. Am J Obstet Gynecol. 1985;152:91-7.

29. ÓNeill ME, Cooper KA, Hunyor SN, Boyce ES. Cardiorespiratory response to walking in trained and sedentary pregnant women. $J$ Sport Med Phys Fit. 1993;33:40-3.
30. Hall P. Cardiovascular adaptations to physical conditioning during pregnancy. Thesis. Kingston: Queen's University; 1987.

31. Melzer K, Schutz Y, Boulvain M, Kayser B. Physical activity and pregnancy cardiovascular adaptations. Recommendations and pregnancy outcomes. Sports Med. 2010;40:493-507.

32. Ihrman K. A clinical and physiological study of pregnancy in a material from Northern Sweden VIII. The effect of physical training during pregnancy circulatory adjustment. Acta Soc Upsal. 1960;65:335-47.

33. VeilleJ-C. Hellerstein HI, Bacevice AE. Maternal left ventricular performance during bicycle exercise. Am J Cardiol. 1992;69: 1506-8.

34. Rubal BJ, Muhailani AR, Rosentswieg J. Effects of physical conditioning on the heart size and wall thickness of college women. Med Sci Sports Exerc. 1987;19:423-9.

35. Veille JC, Hosenpud JD, Morton MJ. Cardiac size and function in pregnancy-induced hypertension. Am J Obstet Gynecol. 1984; 150(5 Pt 1):443-9.

36. Veille JC, Hellerstein HK, Cherry B, Bacevice AE. Effects of advancing pregnancy on left ventricular function during bicycle exercise. Am J Cardiol. 1994;73:609-10.

37. Veille JC. Maternal and fetal cardiovascular response to exercise during pregnancy. Semin Perinatol. 1996;20:250-62.

38. Kaaja RJ, Greer IA. Manifestations of chronic disease during pregnancy. JAMA. 2005;294:2751-7.

39. Panchal S, Arria AM, Labhsetwar SA. Maternal mortality during hospital admission for delivery: A retrospective analysis using a state-maintained database. Anesth Anal. 2001;93: 134-41.

40. Basso $O$, Rasmussen $S$, Weinberg CR, Wilcox AJ, Irgens LM, Skjaerven R. Trends in fetal and infant survival following preeclampsia. JAMA. 2006;296:1357-62.

41. McElrath TF, Hecht JL, Dammann O, Boggess K, Onderdonk A, Markenson G, et al. Pregnancy disorders that lead to delivery before the 28th week of gestation: An epidemiologic approach to classification. Am J Epidemiol. 2008;168:980-9.

42. Redman CW, Sargent IL. Latest advances in understanding preeclampsia. Science. 2005;308:1592-4.

43. Zhang J, Meikle S, Trumble A. Severe maternal morbidity associated with hypertensive disorders in pregnancy in the United States. Hypertension in Pregnancy. 2003;22:203-12.

44. McDonald SD, Malinowski A, Zhou Q, Yusuf S, Devereaux PJ. Cardiovascular sequelae of preeclampsia/eclampsia: A systematic review and meta-analyses. Am Heart J. 2008;156:918-30.

45. anderson $\mathrm{CM}$. Preeclampsia. Exposing future cardiovascular risk in mothers and their children. J Obstet Gynecol Neonatal Nurs. 2007;36:3-8.

46. Kiel D, Dodson E, Artal R, Boehmer T, Leet T. Gestational weight gain and pregnancy outcomes in obese women: how much is enough? Obstet Gynecol. 2007;110:752-8.

47. Lombardi DG, Barton JR, O'Brien JM, Istwan NK, Sibai BM. Does an obese prepregnancy body mass index influence outcome in pregnancies complicated by mild gestational hypertension remote from term? Am J Obstet Gynecol. 2005;192:1472-4.

48. Catalano PM. Obesity, insulin resistance, and pregnancy outcome. Reproduction. 2010;140:365-71.

49. Alaily $A B$, Carrol KB. Pulmonary ventilation in pregnancy. $\mathrm{Br} \mathrm{J}$ Obstet Gynecol. 1978;85:518-24.

50. Evenson KR, Savitz DA, Huston SL. Leisure-time physical activity among pregnant women in the US. Paediatr Perinat Epidemiol. 2004;18:400-7.

51. Schmidt Stutzman S, Brown CA, Hains SM, Godwin M, Smith GN, Parlow JL, et al. The effects of exercise conditioning in normal and overweight pregnant women on blood pressure and heart rate variability. Biol Res Nurs. 2010;12:137-48.

52. Jessurun C, Jessurun A, Adam C, Brown D, Vaughn W, Wilansky S. Left Ventricular Diastolic Function in Normal Human Pregnancy. Circulation. 1999;99:511-7. 